\title{
Modeling and Formulation of a Novel Microoptoelectromechanical Gyroscope
}

\author{
Bohua Sun, Bo Zhang, and Mohamed Toriq Khan \\ Centre for Mechanics, Smart Structure and Micro-Systems, Cape Peninsula University of Technology, \\ Cape Town 7535, South Africa \\ Correspondence should be addressed to Bohua Sun, sunb@cput.ac.za
}

Received 20 February 2008; Accepted 12 May 2008

Recommended by Rakesh Joshi

This paper proposed a novel design of microgyroscope based on MEMS structures and optic interferometric microdisplacement measurement technique. The gyroscope consists of microvibrator and interferometric readout. Using Coriolis force, the vibrator transfers the system rotation into a forced vibration; the induced vibration can be sensed by the interferometric microdisplacement measurement system. The optic measurement system has two mirrors which will reflect two rays into a detector. The comprehensive studies on the formulation and analysis of the proposed gyroscope have been undertaken; two key sensor equations have been derived in the first time in the world: (1) relation between rotation and phase shift of light $\Delta \varphi=\left(4 \pi l_{0} / \lambda\right)+(8 \pi / \lambda)\left(x_{\max } Q_{y} / \omega_{y}\right) \Omega(t) \sin \left(\omega_{d} t\right),(2)$ relation between rotation and interferometric intensity of light $I(t) \approx$ $(8 \pi / \lambda)\left(x_{\max } Q_{y} / \omega_{y}\right) \Omega(t) \sin \left(\omega_{d} t\right) \sin \left(4 \pi l_{0} / \lambda\right)$. The comparison of the proposed gyroscope and well-know Sagnac formulation has been investigated; it shown that the proposed model is much better than Sagnac ones. The new model has finally get rid of needing very long fiber in the case of Sagnac gyroscope. The innovative model gives a new hope to fabricate high accurate and cheaper gyroscope. To date, the proposed gyroscope is the most accurate gyroscope.

Copyright (C) 2008 Bohua Sun et al. This is an open access article distributed under the Creative Commons Attribution License, which permits unrestricted use, distribution, and reproduction in any medium, provided the original work is properly cited.

\section{INTRODUCTION}

Gyroscopes are the inertial measurement devices, which are used to detect angular rotation rate of the objects. which are widely used for guidance, navigation, airplanes, spacecrafts, missiles, automobiles, and even consumer electronics to maintain orientation, measure the angular motion of essential objects for the control and stabilization of its attitude.

The gyroscope development is going through the processes from the macrosize to the microsize. Traditional gyroscopes with spinning wheels employed in the present aerospace and military industries are bulky, which need lubricant and wear out, as stability control system, navigation system, and so forth. Ring laser gyroscopes (RLGs) and fiber optical gyroscopes (FOGs) are high precision. But they are expensive and heavy. The micro applications require the host structures to be transformed from the huge spin wheel mass to the tiny light vibration mass or optical interferometer measurement system fabricated by microelectromechanical system (MEMS) technology like MEMS vibratory gyroscopes and fiber optical gyroscopes [1].
In terms of small size critical, MEMS vibratory gyroscopes are the perfect substitution, especially for microsatellites, microunmanned vehicles, digital cameras, and notebook, PDA, due to their small size. MEMS gyroscopes are also suitable for longtime employ because of low power consumption, and the bulk fabrication processes can produce batch applications. Since the light weight and low power consumption, some small instruments like digital cameras can wear several MEMS gyroscopes and accelerometers for positioning or monitoring its activities either in vehicle or out of vehicle during travel [2]. In fact, some types of sell MEMS gyroscopes have started for dynamic control of automobiles a few years ago. Silicon basement sensing system is providing MEMS gyroscopes by Analog Devices.

Silicon-based MEMS gyroscopes processes may be categorized into bulk micromachining and surface micromachining. A large mass is desired for a gyroscope because thermomechanical noise is inversely proportional to mass weight. Generally, MEMS gyroscopes and bulk micromachined gyroscopes have large mass and relatively large 
readout capacitance or piezoresistive readout. Therefore, most bulk bonding to a separate electronic readout chips (two chips). Two chips gyroscopes are expensive and have a relatively large package size that restricts their applications in consumer electronics regardless of price. In contrast, surface micromachining gyroscopes have small mass and relatively small readout capacitance. The sensors and readout electronics are usually integrated on a single chip to reduce parasitic capacitance and size.

MEMS gyroscopes system can only sense in one axis, but angular rate is requiring a vector, measuring all three-axis rotations. Assembling three gyroscopes together is needed, which will increase the package size, and the complexity of packaging cost. The capacitance readout electronic circuits have the essential shortcoming at the sensitivity, responding time, bandwidth, and so forth. Therefore, the current MEMS gyroscopes only occupy the low and middle sensitivity scales markets.

On the other hand, many intrinsic optical theories are used in the new field for pressure, temperature, acceleration, skin friction sensors, and so forth, and the performances of optical sensors and measurements scale are better than mechanical sensors or others at sensitivity, volumes, cost, stability, and so forth. Optical type gyroscopes have advantages at the sensitivity, performance, responding time, lifetime, and bandwidth. They occupy the high sensitivity and performance markets. Ring laser gyroscopes, fiber optical gyroscopes, and other optical gyroscopes are too large and heavy for many applications due to the Sagnac effect principle, which is the basic theory for all kinds of optical gyroscopes. The large size comes from the long distance light path to create the enough interference and the lack of MEMS fabrication technology for the micro-optical devices.

However, the MEMS inertial sensors have the following advantages compared with the conventional inertial instruments [3].

\section{(1) Smaller volume and lighter weight}

The advanced semiconductor manufacturing processes make the volume smaller and the weight of the microinertial mechanical sensors lighter.

\section{(2) Mass production and lower production cost}

Thousands of microinertial devices in a 3 inch or 4 inch silicon chip could be yielded with the mature technique and batch production, which will improve the rate of finished products and reducing the cost. The cost of microinertial devices, then, will depend on the packaging, testing, and accessory circuits. It is undoubted that the cost would drop greatly when the true integration of electromechanical is accomplished.

\section{(3) Antiharsh environments and wider applications}

With the small testing proof mass, a micromechanical accelerometer is adapted to measure the high acceleration.

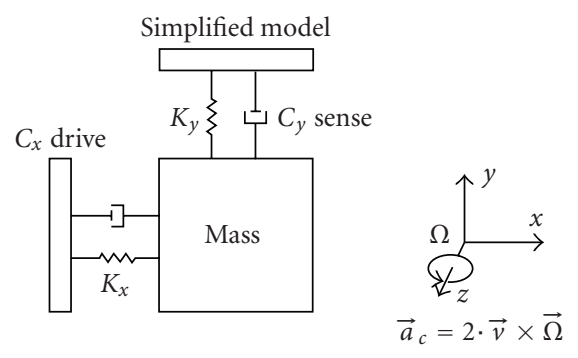

FIGURE 1: Model of coriolis force.

The measurement range could be extended widely by the micromechanical gyroscopes without rotating parts.

\section{(4) Lower consumption, higher sensitivity and efficiency}

When performing the same task, the consumption of micromechanical devices is only one tenth of the traditional ones or even less, while the operation speed could be improved ten times. Certain reasons, for example, the no delay performance of integrated micromechanical devices results in their wide use in high-speed application.

\section{(5) Multifunction and intelligence}

Most micromechanical devices integrated the transducer, actuator, and electronic circuits. It is easier to realize the multifunction and intelligence of micromechanical devices after using the intelligent materials and structures.

The novel combination of the microvibratory gyroscope and optical interferometer readout system is proposed in this paper. The merged system has the small size and high performance and is suitable for MEMS fabrication technology. In this paper, the system design, mathematical model, and performance analysis are presented.

\section{MICROMECHANICAL DESIGN OF THE MOEMS GYROSCOPE}

Based on the comprehensive review of the optical gyroscopes and MEMS silicon-based gyroscopes, we propose a hybrid model which combine MEMS silicon-based oscillation gyroscopes and micro-optical interferometer readout system. It will take both advantages of MEMS vibratory gyros and optical sensing technique. It is believed that the proposed gyroscope can be massively fabricated with high performance at low cost.

The operation of the $z$-axis vibratory rate gyroscope is best illustrated by the spring-mass system shown in Figure 1. As in all vibratory rate gyroscopes, Coriolis acceleration is generated by an oscillating proof mass in a rotating reference frame. The proof mass is oscillated along the $x$-axis (the driven mode), the reference frame rotates around the $z$-axis, and the Coriolis acceleration is detected as deflections along the $y$-axis (the sense mode) [4].

The microelectromechanical sense element of the surface micromachined $z$-axis vibratory rate gyro is also shown in Figure 2. The structure comprises three major elements: 


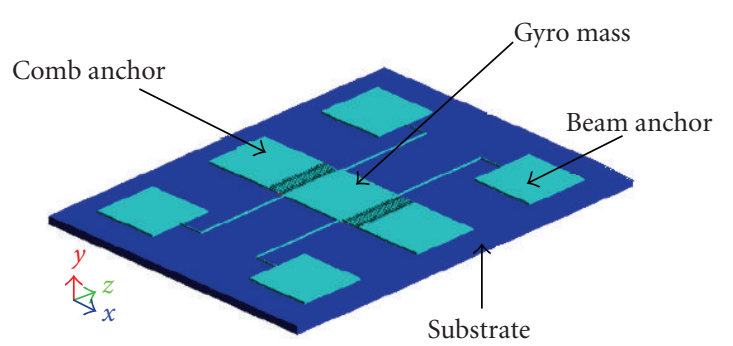

FIGURE 2: Solid model of MEMS vibratory structure.

the suspension system, the comb drive used to sustain oscillation, and the proof mass forced by the Coriolis force in $y$-axis.

Coriolis acceleration is

$$
\vec{a}_{c}=2 \cdot \vec{v} \times \vec{\Omega}
$$

which shows that orthogonal lateral-axis vibration and acceleration sensing are required to detect the $z$-axis rotation. The mathematics principle model is shown in Figure 1.

One vibration mode corresponds to the vibration of mass in the $x$-direction with a vibration frequency of $\omega_{x}$. The other vibration corresponds to the vibration of mass in the $y$-direction with a vibration frequency of $\omega_{y}[5,6]$.

For operation, the mass is driven into vibration in the $x$ direction with a driving frequency $\omega_{d}$.

Given driven signal,

$$
x=A_{d} \sin \omega_{d} t
$$

Then, if the system rotates around the $z$-axis with a constant angular rate of $\Omega$, the Coriolis force appears in the $y$-axis and impelling the mass vibrating in $y$-axis:

$$
\begin{aligned}
\dot{x} & =A_{d} \omega_{d} \cos \omega_{d} t, \\
F_{c} & =2 m \dot{x} \Omega .
\end{aligned}
$$

Differential equation for the mass movement in the $y$ direction is given by

$$
m \ddot{y}+c_{y} \dot{y}+k_{y} y=2 m A_{d} \omega_{d} \Omega \sin \left(\omega_{d} t+\frac{\pi}{2}\right) .
$$

The steady-state solution is given by

$$
y=A_{y} \sin \left(\omega_{d} t+\frac{\pi}{2}-\varphi\right)=A_{y} \cos \left(\omega_{d} t-\varphi\right) .
$$

There, amplitude at $y$-direction is

$$
A_{y}=\frac{2 A_{d} \omega_{d} \Omega}{\omega_{y}^{2} \sqrt{\left(1-\omega_{d}^{2} / \omega_{y}^{2}\right)^{2}+4 \zeta_{y}^{2}\left(\omega_{d}^{2} / \omega_{y}^{2}\right)}} .
$$

Phase is

$$
\begin{aligned}
& \varphi=\tan ^{-1} \frac{2 \zeta_{y} \omega_{d} \omega_{y}}{\omega_{y}^{2}-\omega_{d}^{2}}, \\
& \zeta_{y}=\frac{c_{y}}{2 m \omega_{y}},
\end{aligned}
$$

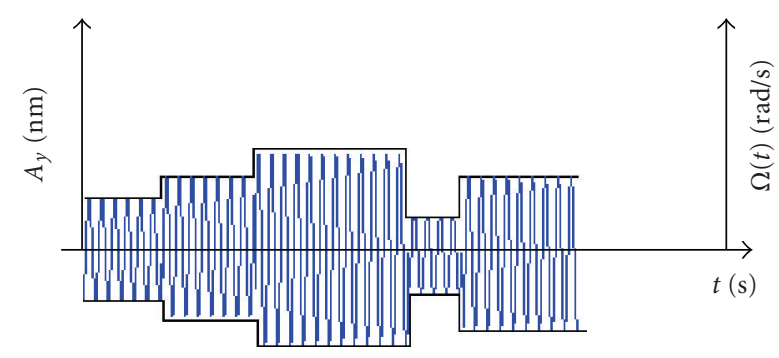

$$
\begin{aligned}
& -\Omega(t) \\
& -A_{y}
\end{aligned}
$$

FIGURE 3: Oscillating coriolis displacements creating by variable rotation velocity.

where $Q_{y}$ is the quality factor at the sensing mode given by

$$
Q_{y}=\frac{1}{2 \zeta_{y}} \text {. }
$$

Let $\omega_{d}=\omega_{y}$, the mass amplitude becomes at resonance

$$
A_{y}=\frac{2 A_{d} \Omega Q_{y}}{\omega_{y}}=\frac{2 A_{d} Q_{y}}{\omega_{y}} \Omega .
$$

Equation (9) shows that the measurement of rotation $\Omega$ can be achieved by sensing the amplitude of vibration in $y$ direction, the problem of measurement of rotation becomes a problem of sensing a displacement. It is the foundation of vibratory gyroscope principle.

The system response froced Coriolis acceleration along $y$-axis is given by (9).

The vibration along $y$-axis is

$$
\frac{y}{\Omega}=\frac{2 A_{d} Q_{y}}{\omega_{y}} \sin \left(\omega_{d} t+\frac{\pi}{2}-\varphi\right) .
$$

The results of the relative between the angular rate and Coriolis acceleration are clear, which shows the $y$-axis amplitude proportion with the $z$-axis angular rate, and the $x$-axis driver oscillation redound to amplifier the amplitude of the $y$-axis [7]. In the microsize, the nature frequency of oscillation beams and proof mass is very high, and the weight is very light. It is of the advantage driving the mass at the $x$ axis under low power consumption, and achieves the high sensitivity.

Now, the angular rate measurements convert to measure the $y$-axis amplitude, shown in Figure 3. This changing gives the measurement idea, which is microdisplacement measurement. There are many methods to measure microdisplacements like bridge resistance, bridge capacitance, piezoelectricity, optical modulation methods, and so forth. Currently, the optical interferometer is the most precise method, which is the method proposed in this paper.

\section{MICRO-OPTICAL INTERFEROMETRIC DESIGN OF THE MOEMS GYROSCOPE}

From (9), we know that the problem of measurement of rotation becomes a problem of sensing a displacement. There 


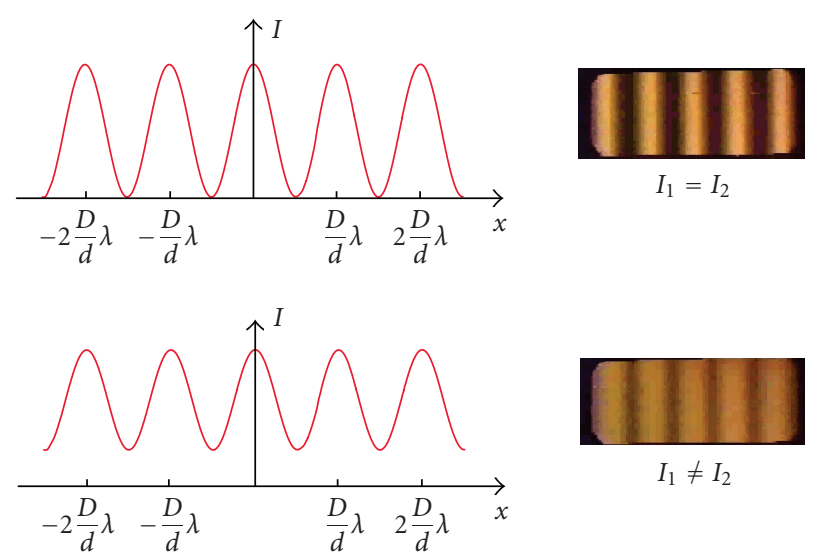

FIGURE 4: Interferometer output intensity and patterns.

are several ways to sense the displacement, but up to now the optic sensing is the most accurate one.

The optical interferometric readout system has the high sensitivity, high precision, high integration, low cost characteristics. Interference, which is a complex wave phenomenon, is one of the useful readout, which can be happened and observed in frequency wave areas, like the mechanical wave, sound wave, light wave, electric wave, and so forth [8]. In the optical technology, the interference will be happened under those situations, no matter the sensor is a Michelson, a Mach-Zehnder, a Sagnac, or a Fabry-Perot interferometer, (1) frequency of two beams must be same; (2) the vibration of two beams must be same direction; (3) phase shift of two beams must be holed constant.

The intensity of general interferometer output is composition of two light beams $I_{1}$ and $I_{2}$ :

$$
I=I_{1}+I_{2}+2 \sqrt{I_{1} I_{2}} \cos \varphi_{12}
$$

Here, $\varphi_{12}$ is the phase shift different of two beams at interaction point:

$$
\varphi_{12}=\varphi_{1}-\varphi_{2}-2 \pi \frac{\Delta L}{\lambda}
$$

Here $\varphi_{1}$ and $\varphi_{2}$ are initialization phase of two beams. $\Delta L$ is the path-length different of two beams.

The periodic intensity patterns are shown in Figure 4 .

The phenomenon of the interference of light underlies many high-precision measuring systems and displacement sensors. The use of optical fibers allows making such devices extremely compact and economic. In fiber optic interferometer, the interference occurs at the partially reflecting end face surface of the fiber and an external mirror. The size of the sensitive element based on this principle can be as small as diameter of the fiber, and the sensitivity can achieve subangstrom level. Additionally, such sensor is not sensitive to electromagnetic interference and can be used in hostile environment.

Consider the principle of operation of the fiber optic interferometer, the system view shown in Figure 5, and the magnifying sensor head configurations shown in Figure 6.

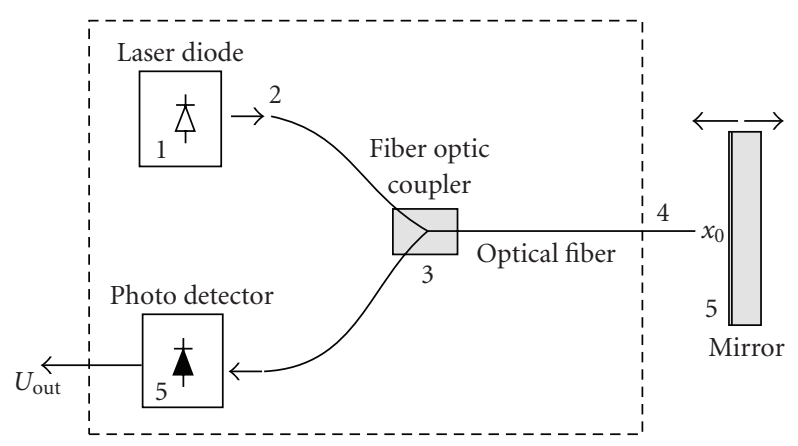

FIGURE 5: Diagram of micro fiber optical interferometer.

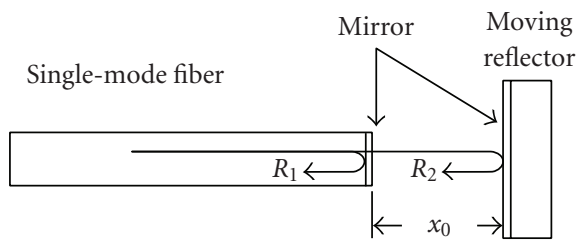

Figure 6: Magnify fiber optic interferometer head.

The radiation of the laser diode 1 is coupled into fiber 2 and propagates through coupler 3 to fiber 4 . Then, one part of radiation is reflected from the end face of fiber 4 , and other part of radiation is flashed into the air, reflected from the mirror 5 and returned back into fiber 4 . The optical beam reflected from the end face of fiber 4 interferes with the beam reflected from the mirror. As a result, the intensity of the optical radiation at photodetector 5 is periodically changed depending on the distance $x_{0}$ between the fibers and mirrors as follows. The path-length difference is $\Delta L=2 x_{0}$.

The out intensity is

$$
I=I_{1}+I_{2}+2 \sqrt{I_{1} I_{2}} \cos \left(\frac{4 \pi}{\lambda} x_{0}+\varphi_{0}\right)
$$

Here, $\varphi_{0}$ is initialization phase shift. $\lambda$ is the wavelength of the light source used; phase shift is $\Delta \varphi=(2 \pi / \lambda) \Delta L=$ $(4 \pi / \lambda) x_{0} ; I_{1}$ and $I_{2}$ are intensities of these two reflected rays.

In fiber optic interferometer, $I_{1}=I_{0} R_{1}$ is the intensity of the light reflected from the end face surface of the fiber, and $I_{2}=\left(1-R_{1}\right)^{2} R_{2} I_{0}$ is the intensity of the light reflected from an external mirror and returned back into the fiber, where $I_{0}$ is the intensity of the laser diode radiation coupled into the fiber, $R_{1}$ is the reflectivity of the end face of the fiber, and $R_{2}$ is the reflectivity of an external mirror. Figure 7 shows the simulation result of the relationship between sensor output $I_{0} / I$ and gap distance $x_{0}$. This gap distance $x_{0}$ is the position to setup the movement reflected surface, which is also the definition of the interferometer cavity length $[9,10]$ :

$$
I=I_{0}\left[R_{1}+\left(1-R_{1}\right)^{2} R_{2}+2\left(1-R_{1}\right) \sqrt{R_{1} R_{2}} \cos \left(\frac{4 \pi}{\lambda} x_{0}+\varphi_{0}\right)\right],
$$




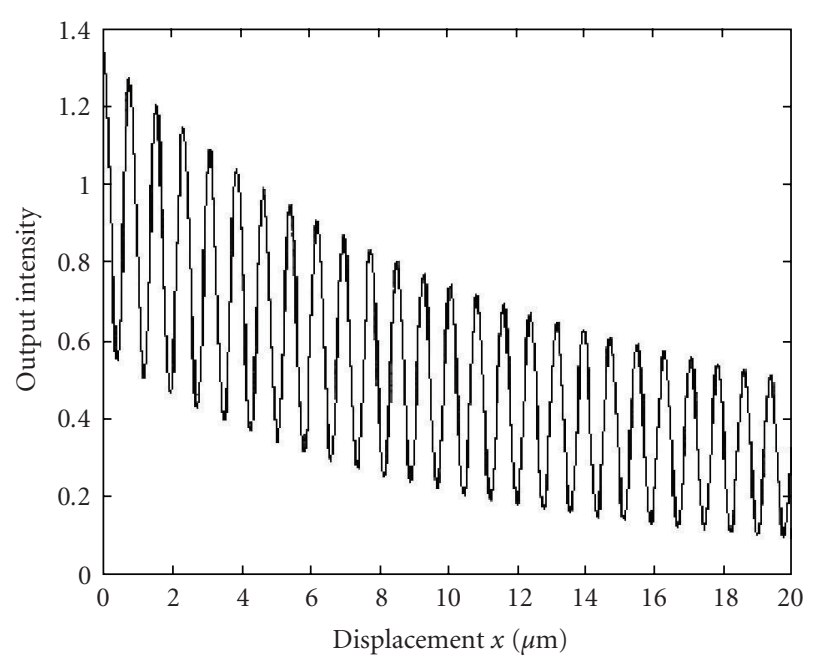

FIgURE 7: Simulation result of the relationship between sensor output $I_{0} / I$ and gap distance $x$.

in which $I_{1}=I_{0} R_{1}$ and $I_{2}=\left(1-R_{1}\right)^{2} R_{2} I_{0}$ are constant, by applying demodulation technique, the above equation (14) can be simplified as

$$
I \approx \cos \left(\frac{4 \pi}{\lambda} x_{0}+\varphi_{0}\right)
$$

For simplification and cavity design purpose, let $x_{0}=k \lambda / 2$, and $\varphi_{0}=0$, then we have

$$
\begin{aligned}
\Delta \varphi & =\frac{4 \pi}{\lambda} x_{0}=2 k \pi, \quad k=0, \pm 1, \pm 2, \ldots, \\
I_{\max } & =\left(\sqrt{I_{o} R_{1}}+\sqrt{\left(1-R_{1}\right)^{2} R_{2} I_{o}}\right)^{2} .
\end{aligned}
$$

Let $x_{0}=(\lambda / 4)(2 k+1)$,

$$
\begin{gathered}
\Delta \varphi=\frac{4 \pi}{\lambda} x_{0}=(2 k+1) \pi, \quad k=0, \pm 1, \pm 2, \ldots, \\
I_{\min }=\left(\sqrt{I_{o} R_{1}}-\sqrt{\left(1-R_{1}\right)^{2} R_{2} I_{o}}\right)^{2} .
\end{gathered}
$$

From the above intensity distributing analysis, as every time the cavity length $x_{0}$ is changing $\lambda / 4$, the intensity would be varied from maxima to minima.

So the movement distance can be calculated by

$$
\Delta S=\frac{\lambda}{4} N
$$

Here, $\Delta S$ is the distance moving from initialization position $x_{0} . N$ is the times of the intensity changing.

Let $\varphi_{0}^{\prime}=(4 \pi / \lambda) x_{0}$ be presenting cavity $x_{0}$ equivalent initialization phase shift. The initialization phase $\varphi_{0}^{\prime}$ is located at the middle of $I_{\max }$ and $I_{\min }$, because here is maxima linear slope, shown in Figure 8. For microdisplacement, the accurate output result can be achieved in this linear region. The initialization position for movement reflected surface is

$$
\begin{aligned}
x_{0} & =\frac{k \lambda}{8}, \\
I_{x_{0}} & =\frac{I_{\max }-I_{\min }}{2} .
\end{aligned}
$$

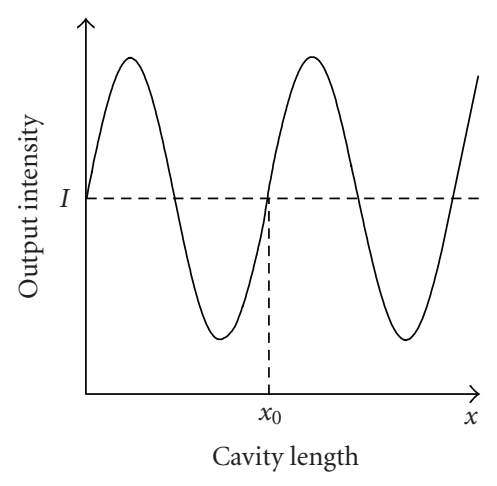

FIGURE 8: Initialization position of the vibratory reflected surface and linear region.

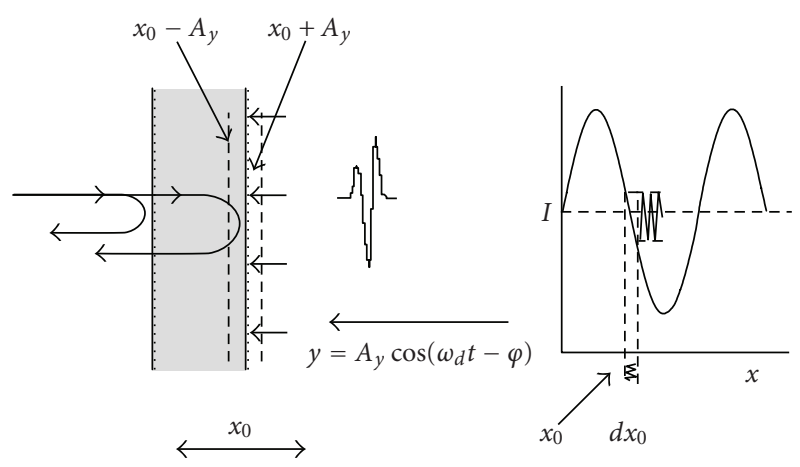

FIGURE 9: Effecting oscillation input to output intensity.

Now, we will consider the interferometric signal appearing as a result of the reflection of the light from the vibrating surface (resonator). When the resonator oscillates, the phase difference of interfering rays is varied as follows. The principle diagram shown in Figure 9 is describing the affection oscillation input to output intensity of the microfiber optical interferometer. The distance measurement performance of the micro-optical fiber interferometer is proved under $5 \mathrm{~nm}$ $[11,12]$.

According to the Coriolis acceleration theory, the $y$-axis vibration created by $z$-axis ration rate is

$$
y=A_{y} \sin \left(\omega_{d} t+\frac{\pi}{2}-\varphi\right)=A_{y} \cos \left(\omega_{d} t-\varphi\right) .
$$

Path-length difference is

$$
\begin{aligned}
\Delta L & =2\left[x_{0}+A_{y} \cos \left(\omega_{d} t-\varphi\right)\right], \\
\Delta \varphi & =\frac{4 \pi}{\lambda}\left[x_{0}+A_{y} \cos \left(\omega_{d} t-\varphi\right)\right] .
\end{aligned}
$$

This $A_{y} \cos \left(\omega_{d} t-\varphi\right)$ vibration signal gives rise to the following modulation of the light intensity:

$$
\begin{aligned}
I(t) & \cong \cos \left\{\frac{4 \pi}{\lambda}\left[x_{0}+A_{y} \cos \left(\omega_{d} t-\varphi\right)\right]\right\} \\
& \cong \cos \left[\frac{4 \pi}{\lambda} A_{y} \cos \left(\omega_{d} t-\varphi\right)+\frac{4 \pi}{\lambda} x_{0}\right] .
\end{aligned}
$$




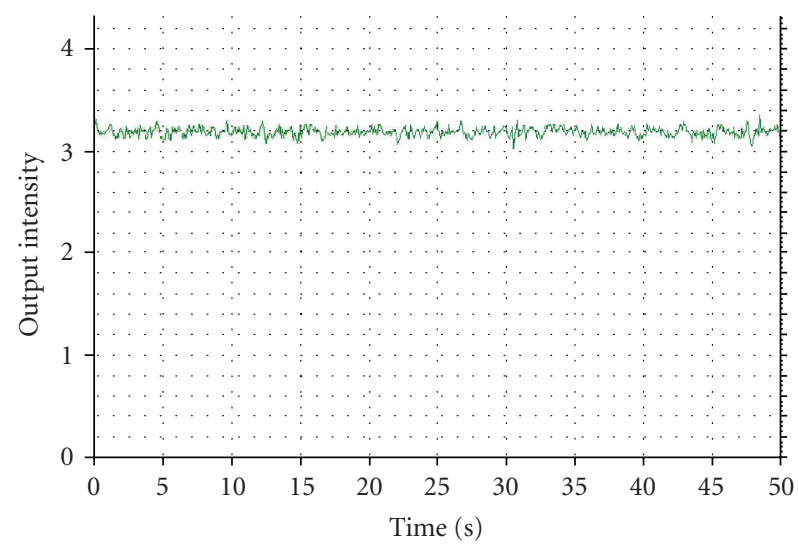

FIgURE 10: Output intensity at $\Omega=0$.

Expanding $I(t)$ in a Fourier series, and Bessel function $\cos (x \sin y)=J_{0}(x)+2 \sum_{n=1}^{\infty} J_{2 n}(x) \cos 2 n y$, the alternating components of the light modulation is:

$$
\begin{aligned}
I(t) \cong & J_{1}\left(\varphi_{\omega}\right) \sin (\omega t-\eta) \sin \varphi_{0}^{\prime} \\
& -J_{2}\left(\varphi_{\omega}\right) \cos (\omega t-\eta) \cos \varphi_{0}^{\prime}+\cdots,
\end{aligned}
$$

where $J_{i}\left(\varphi_{\omega}\right)$ is the Bessel functions, $\varphi_{\omega}=(4 \pi / \lambda) A_{y}$. When $\varphi_{\omega} \ll 1$, then $J_{i}\left(\varphi_{\omega}\right)$ equals approximately $\varphi_{\omega} / 2$ and, therefore, an alternating component of intensity $I(t)$ will be proportional to displacement of the resonator from the equilibrium: $I(t) \sim \sin (\omega t):$

$$
\begin{aligned}
I(t) & \cong \frac{1}{2} \varphi_{\omega} \sin (\omega t-\eta) \sin \varphi_{0}^{\prime} \\
& \cong \frac{2 \pi}{\lambda} A_{y} \sin \varphi_{0}^{\prime} \cos \left(\omega_{d} t-\varphi\right) .
\end{aligned}
$$

Let $\omega_{d}=\omega_{y}$, the amplitude becomes

$$
\begin{aligned}
A_{y} & =\frac{2 A_{d} \Omega Q_{y}}{\omega_{y}}=\frac{2 A_{d} Q_{y}}{\omega_{y}} \Omega, \\
I(t) & \cong \frac{4 \pi A_{d} Q_{y}}{\lambda \omega_{y}} \Omega \sin \varphi_{0}^{\prime} \sin \left(\omega_{d} t+\frac{\pi}{2}-\varphi\right), \\
\Omega & \cong \frac{I(t) \lambda \omega_{y}}{4 \pi A_{d} Q_{y} \sin \varphi_{0}^{\prime} \sin \left(\omega_{d} t+(\pi / 2)-\varphi\right)} .
\end{aligned}
$$

Let $\alpha=\lambda \omega_{y} / 4 \pi A_{d} Q_{y} \sin \varphi_{0}^{\prime}$,

$$
\Omega \cong \alpha \frac{I(t)}{\cos \left(\omega_{d} t-\varphi\right)}=\alpha I(t) \cos ^{-1}\left(\omega_{d} t-\varphi\right) .
$$

The linear displacement linked between the $y$-axis vibration in MEMS structure and the optical readout system is the key factor for the microopticelectromechanical gyroscope; the system model is in Figure 12. The vibration parameter is the requirement measurement, which is creating the intensity changing as well.

The relative parameters of the angular motion can be calculated by integrating (27). This results in the equation

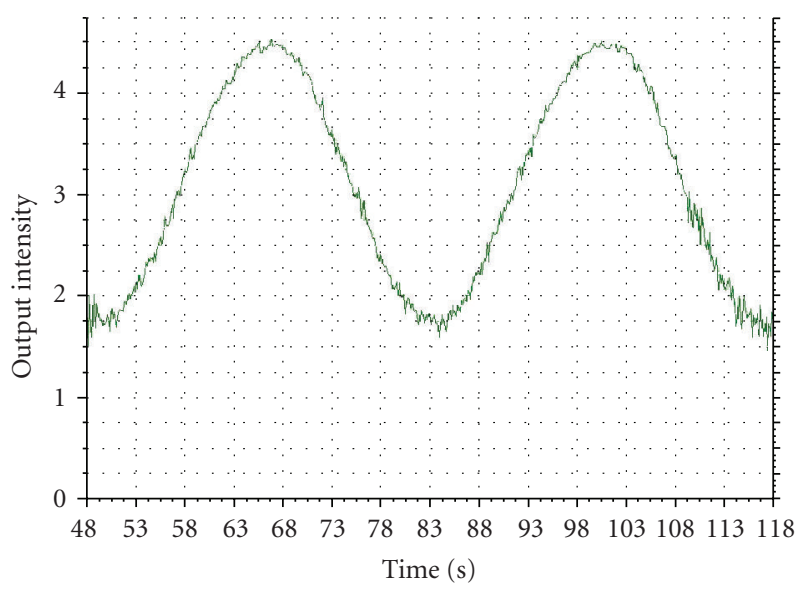

FIGURE 11: Output intensity at $\Omega \neq 0 \mathrm{rad} / \mathrm{s}$.
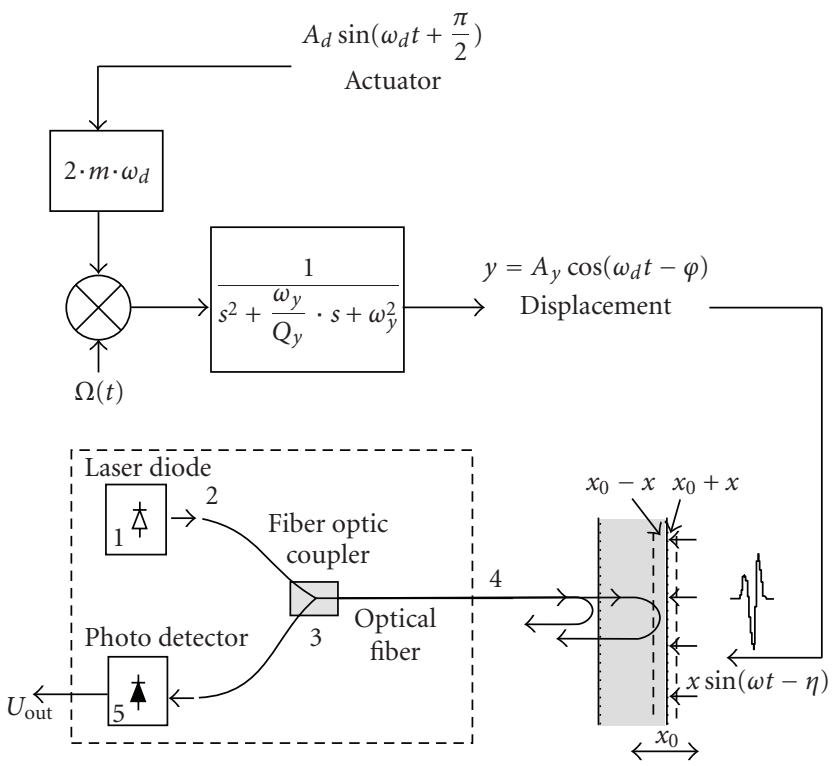

FIGURE 12: Model of micro vibratory optical readout gyroscope system.

below indicate angular displacement, $\theta$, which is useful for navigation systems directly.

The angular movement is the integral of angular rate:

$$
\begin{aligned}
\Delta \theta & =\int \Omega(t) d t=\alpha \int_{t 1}^{t 2} \frac{I(t)}{\cos \left(\omega_{d}-\varphi\right)} d t \\
& =\left[\alpha \frac{1}{\omega_{d}} \ln \left|\tan \left(\frac{\omega_{d} t}{2}+\frac{\pi}{4}\right)\right|+C\right] \int_{t 1}^{t 2} I(t) d t .
\end{aligned}
$$

From (29), the operator of navigation would indicate the angular movement variation value $\Delta \theta=\theta_{t 2}-\theta_{t 1}$; feedback signal would emendate the application during the steering.

Therefore for any intensity readout, the angular movement value can be calculated as $\Delta \theta$ due to the reference frame, by means of a simple digital signal processor circuit attached to the optical readout of the MOEMS device. 


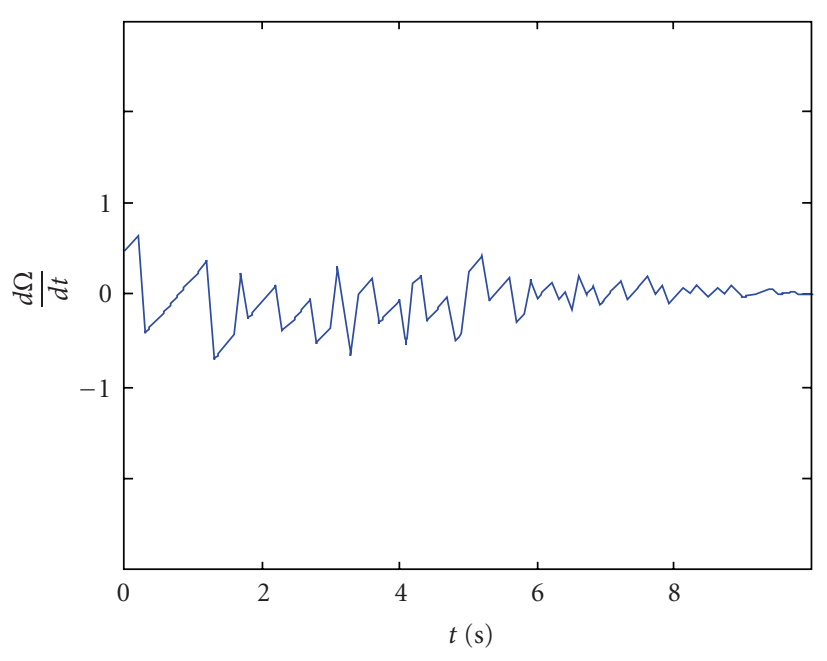

FIgURE 13: Angular acceleration attenuation diagram.

If (27) is differentiated, the rotation acceleration is obtained, shown in

$$
\ddot{\theta}=\frac{d \Omega}{d t}=\alpha \frac{\dot{I}(t)+I(t) \omega_{d} \tan \left(\omega_{d} t-\varphi\right)}{\cos \left(\omega_{d} t-\varphi\right)} .
$$

This is another useful output signal to control the system's stability. From (30), the signal is fast attenuation signal as the expectation. The application is illustrated in Figure 13.

\section{MOEMS GYROSCOPE COMPARISON WITH OPTICAL GYROSCOPE BASED ON SAGNAC EFFECT}

The Sagnac effect is the relative phase shift between two beams of light that have traveled an identical path in opposite direction in a rotating frame. The explanation of the Sagnac effect is simple for the inertial frame of reference. The motions of the mirrors, during the light transit time between mirrors, cause the clockwise and counterclockwise waves to be reflected at different points of space, which leads to an optical path difference. Modern fiber-optic gyroscopes (Sagnac interferometers) used for navigation are based on this effect. They allow highly accurate measurements of rotation rates down to about $0.1^{\circ}$ per hour. The schematic setup of a Sagnac interferometer is shown in Figure 14. Light is decomposed into two beams by a 50:50 beam splitter, with one traveling clockwise (CW) and the other counterclockwise (CCW) around a polarization-maintaining single-mode glass-fiber loop. The two beams interfere after passage through the loop, and the interference signal is measured with a photodiode. If only reciprocal effects are involved in the experiment, then the two beams interfere constructively (relative phase shift $\Delta \phi=0$ ). If $\Delta \phi \neq 0$, then nonreciprocal effects occur, one of them is the Sagnac effect that results from rotation of the fiber loop during the measurement.

The Sagnac effect is a relativistic effect but can be understood with a simple picture (see Figure 15). Let us assume that a fiber coil ( $N$ windings with radius $R$ ) is rotated

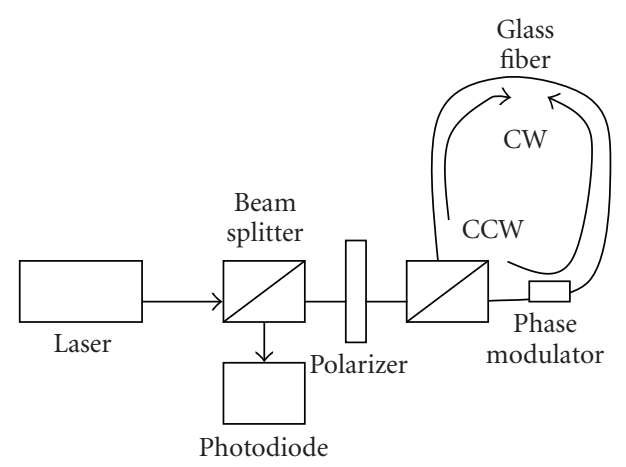

FIGURE 14: Schematic setup of a Sagnac interferometer.

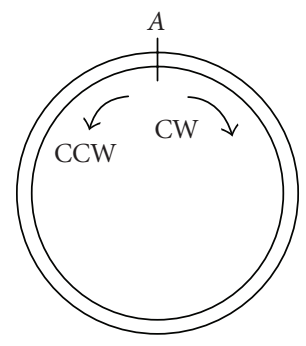

(a)

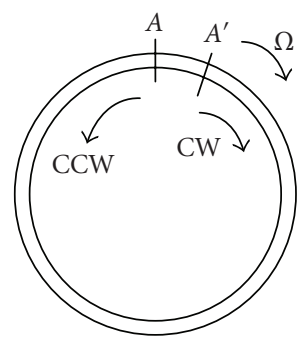

(b)
FIgURE 15: The Sagnac effect principle diagram.

clockwise with an angular velocity, and that light is injected into the loop at time $t=0$. At $t_{t} \approx 2 \pi R N / c$, the CW and CCW beams meet again at the starting point. However, due to the rotation of the loop, they have traveled in different path lengths. Figure 15 on the right indicates what happen if the loop itself is rotating during this procedure. Supposing that radius of fiber round is $R$, and light emitting and detector sensors are laid in " $A$ " point. The system is rotating with $\Omega$ in the clockwise direction relatively inertia space. When clockwise direction light (CW) and the counter-clockwise direction light (CCW) are emitted in opposite direction around, the photo sensor also rotates from " $A$ " to " $A$." So the two light paths have different length. The clockwise direction light pursues " $A$ " after back, which crosses the distance more than $2 \pi R$. While the counterclockwise direction light goes face to " $A$," which crosses the distance less than $2 \pi R$. The difference between the travel times causes the difference light distance [13].

Assume light transmitting in vacuum, which velocity is $c=3 \times 10^{8}$. The paths of clockwise and counterclockwise light are $L_{\mathrm{CW}}, L_{\mathrm{CCW}}$; spending time is $t_{\mathrm{CW}}, t_{\mathrm{CCW}}$, individually.

$$
\begin{aligned}
& \text { Let } C=C_{\mathrm{cw}}=C_{\mathrm{ccw}}, \\
& \qquad \begin{aligned}
L_{\mathrm{cw}} & =2 \pi R+R \Omega t_{\mathrm{cw}}=C_{\mathrm{cw}} t_{\mathrm{cw}}, \\
L_{\mathrm{ccw}} & =2 \pi R-R \Omega t_{\mathrm{cw}}=C_{\mathrm{ccw}} t_{\mathrm{ccw}} .
\end{aligned}
\end{aligned}
$$


From (1), $\Delta t$ is

$$
\begin{aligned}
\Delta t & =t_{\mathrm{cw}}-t_{\mathrm{ccw}}=2 \pi R \frac{2 \pi \Omega-\left(C_{\mathrm{cw}}-C_{\mathrm{ccw}}\right)}{C_{\mathrm{cw}} \cdot C_{\mathrm{ccw}}} \\
& =2 \pi R \frac{2 \pi R \Omega}{C^{2}}=\frac{4 A \Omega}{C^{2}}
\end{aligned}
$$

where $A$ is area of ring fiber round.

Accounting, it is only approximate and simple evolvement above result in (32). The strict evolvement should be applied in broad theory of relativity. The speed of light transmitted in fiber optical depends on refractive index of medium.

So, clockwise and counterclockwise light is

$$
\begin{aligned}
C_{\mathrm{cw}} & =\frac{c / n+R \Omega}{1+((c / n) \cdot R \Omega) / c^{2}}=\frac{c / n+R \Omega}{1+R \Omega / c n}, \\
C_{\mathrm{ccw}} & =\frac{c / n-R \Omega}{1-((c / n) \cdot R \Omega) / c^{2}}=\frac{c / n-R \Omega}{1-R \Omega / c n},
\end{aligned}
$$

where $n$ is refractive index of medium. From (33), we have

$$
\begin{aligned}
\Delta t & =t_{\mathrm{cw}}-t_{\mathrm{ccw}}=2 \pi R \frac{2 \pi \Omega-\left(C_{\mathrm{cw}}-C_{\mathrm{ccw}}\right)}{C_{\mathrm{cw}} \cdot C_{\mathrm{ccw}}} \\
& \approx 2 \pi R \frac{2 R \Omega-2 R \Omega\left(1-\left(1 / n^{2}\right)\right)}{c^{2} / n^{2}}=2 \pi R \frac{2 \pi R \Omega}{C^{2}} \\
& =\frac{4 A \Omega}{C^{2}} .
\end{aligned}
$$

Equation (32) is same as (34) in the vacuum. Corresponding phase difference is

$$
\Delta \phi=\frac{2 \pi \Delta t c}{\lambda}=\frac{8 \pi A \Omega}{\lambda c} .
$$

The difference in path length can be expressed as

$$
\Delta L=2 R \Omega t=\frac{4 \pi R^{2} N}{c} \Omega,
$$

and the corresponding phase difference between the two beams is

$$
\begin{aligned}
\Delta \phi & =\frac{8 \pi^{2} R^{2} N}{\lambda c} \Omega, \\
\Delta \phi_{\text {Sagnac }} & =\frac{8 \pi A}{c \lambda} \Omega, \quad A=\pi R^{2} .
\end{aligned}
$$

It is interesting to note that although the above calculation is over-simplified (e.g., the speed of light in vacuum was assumed instead of that in the glass fiber), it yields the correct result. Exact relativistic calculations show that the phase shift is indeed independent of the material of the wave guide and the above equations apply.

Since the Sagnac effect was discovered, this theory is applied to manufacture the whole optical gyroscope such as laser gyroscope, ring gyroscope, fiber optical gyroscope, and so forth. The fiber optical gyroscope systems are hasty developed due to many advantages like no movement parts inside, high precision output, long consumption, and so forth.
The Sagnac effect method is based on two light beams path-length different created by rotation speed. The long distance light path is required because of the highlight speed. So the disadvantages of Sagnac effect gyroscope system are large in size and weight, complex fabrication processes due to long fiber optical or optical integrated chip.

As mentioned above, in MOEMS gyroscope the light phase shift created by rotation velocity is described

$$
\Delta \phi_{\text {MOEMS_Gyro }}=\frac{4 \pi}{\lambda} l_{0}+\frac{4 \pi}{\lambda} \frac{2 x_{\max } Q_{y}}{\omega_{y}} \Omega \sin \left(\omega_{d} t\right) .
$$

Because $(4 \pi / \lambda) l_{0}$ is constant number, which is defined by initialization interferometer cavity length,

$$
\Delta \phi_{\text {MOEMS_Gyro }}-\frac{4 \pi}{\lambda} l_{0}=\frac{8 \pi}{\lambda} \frac{x_{\max } Q_{y}}{\omega_{y}} \Omega \sin \left(\omega_{d} t\right) .
$$

Assume, at the same input rotation velocity for each gyroscope system, that two-phase shift ratios are

$$
\begin{gathered}
\frac{\Delta \phi_{\text {MOEMS_Gyro }}-(4 \pi / \lambda) l_{0}}{\Delta \phi_{\text {Sagnac }}}=\frac{8 \pi}{\lambda} \frac{x_{\max } Q_{y}}{\omega_{y}} \Omega \sin \left(\omega_{d} t\right) / \frac{8 \pi A}{c \lambda} \Omega, \\
\left|\Delta \phi_{\text {MOEMS_Gyro }}\right|=\left|\left(\frac{x_{\max } Q_{y}}{\omega_{y}} \frac{c}{A}\right) \Delta \phi_{\text {Sagnac }}\right|+\frac{4 \pi}{\lambda} l_{0} .
\end{gathered}
$$

Here $\left(x_{\max } Q_{y} / \omega_{y}\right)(c / A) \geq 1$, which means the sensitivity of MOEMS gyroscope system, is higher than optical gyroscope using Sagnac effect method. So the MOEMS gyroscope design is satisfied to civilization and military user.

\section{CONCLUSION}

In this paper, the combination of the micro-optical interferometer readout system and MEMS vibratory gyroscope is proposed. This novel micro-optical gyroscope has possessed microsize, light weight, and high performance features due to MEMS fabrication technology and the precision feature of the optical interferometer principle. According to comparison with Sagnac effect, the sensitivity and measurement range of this MOEMS gyroscope system are at the same level as the FOG system. The real-time testing results for the MOEMS gyroscope must wait for the first prototype coming out. The full performance comparisons between the MOEMS and FOG system will be investigated.

The novel optical MEMS gyroscope system is the perfect MOEMS application to combine the optical and MEMS technology. On the size aspect, the MEMS vibratory structure has been proved and fabricated to convert the rotation speed to the vibration movement due to Coriolis force theory. On the performance aspect, the microfiber optical interferometer has solved the precision distance readout comparing with the electrical readout. The novel optical MEMS gyroscope can be used from the rate grade to the inertial grade for the pose stability control in the missile, satellite, motion detector in the entertainment system like PC game, camera, or hard drive protector.

The operation principles of the MOEMS gyro system have been clearly discussed and created; the mathematics 
model successfully includes the theory and equations. The MEMS fabrication processes and package for both the microvibration part and the micro-optical readout system will be discussed in the other paper. The first prototype has been sent to MEMS foundry after the software simulating.

\section{ACKNOWLEDGMENT}

The supports from South Africa National Research Foundation and AMSCOR are acknowledged.

\section{REFERENCES}

[1] N. Yazdi, F. Ayazi, and K. Najafi, "Micromachined inertial sensors," Proceedings of the IEEE, vol. 86, no. 8, pp. 1640-1658, 1998.

[2] F Ayazi and K. Najafi, "Design and fabrication of a highperformance polysilicon vibrating ring gyroscope," in Proceedings of the 11th IEEE Annual International Workshop on Micro Electro Mechanical Systems (MEMS '98), pp. 621-626, Heidelberg, Germany, January 1998.

[3] D. Cho, S. Lee, and S. Park, "Surface/bulk micromachined high performance silicon micro-gyroscope," in Proceedings of the Solid-State Sensor Actuator Workshop, Hilton Head Island, SC, USA, June 2000.

[4] D. Kristiansen and O. Egeland, "Modeling of nonlinear vibrations for analysis and control of cylinder gyroscopes," in Proceedings of the 37th IEEE Conference on Decision and Control (CDC '98), vol. 4, pp. 4326-4327, Tampa, Fla, USA, December 1998.

[5] T. Juneau, A. P. Pisano, and J. H. Smith, "Dual axis operation of a micromachined rate gyroscope," in Proceedings of the International Conference on Solid-State Sensors and Actuators (Transducers '97), vol. 2, pp. 883-886, Chicago, Ill, USA, June 1997.

[6] J. Bernstein, S. Cho, A. T. King, A. Kourepenis, P. Maciel, and M. Weinberg, "A micromachined comb-drive tuning fork rate gyroscope," in Proceedings of the IEEE Annual International Workshop on Micro Electro Mechanical Systems (MEMS '93), pp. 143-148, Fort Lauderdale, Fla, USA, February 1993.

[7] X. Jiang, J. I. Seeger, M. Kraft, and B. E. Boser, "A monolithic surface micromachined Z-axis gyroscope with digital output," in Proceedings of IEEE Symposium on VLSI Circuits (VLSI '00), pp. 16-19, Honolulu, Hawaii, USA, June 2000.

[8] W. Ecke, P. Pfeifer, J. Schauer, R. Willsch, and K.-H. Jackel, "Fiber optic displacement measuring system for hightemperature seismic sensor," in Fiber Optic and Laser Sensors XIV, vol. 2839 of Proceedings of SPIE, pp. 290-301, Denver, Colo, USA, August 1996.

[9] "Fiber Optic Interferometer Fabry-Perot," http://www.physics .nad.ru/sensors/English/interf.htm.

[10] E. J. Eklund and A. M. Shkel, "Factors affecting the performance of micromachined sensors based on fabry-perot interferometry," Journal of Micromechanics and Microengineering, vol. 15, no. 9, pp. 1770-1776, 2005.

[11] E. Udd, "Fiber optic smart structures," Proceedings of the IEEE, vol. 84, no. 6, pp. 884-894, 1996.

[12] D. Hofstetter, H. P. Zappe, and R. Dandliker, "Optical displacement measurement with GaAsrAlGaAs-based monolithic integrated Michelson interferometers," IEEE Journal of Lightwave Technology, vol. 15, no. 4, pp. 663-670, 1997.
[13] H. Lefevre, "Principle of the fiber-optical gyroscope," in The Fiber Optic Gyroscope, chapter 2, pp. 5-72, Artech House, Boston, Mass, USA, 1993. 

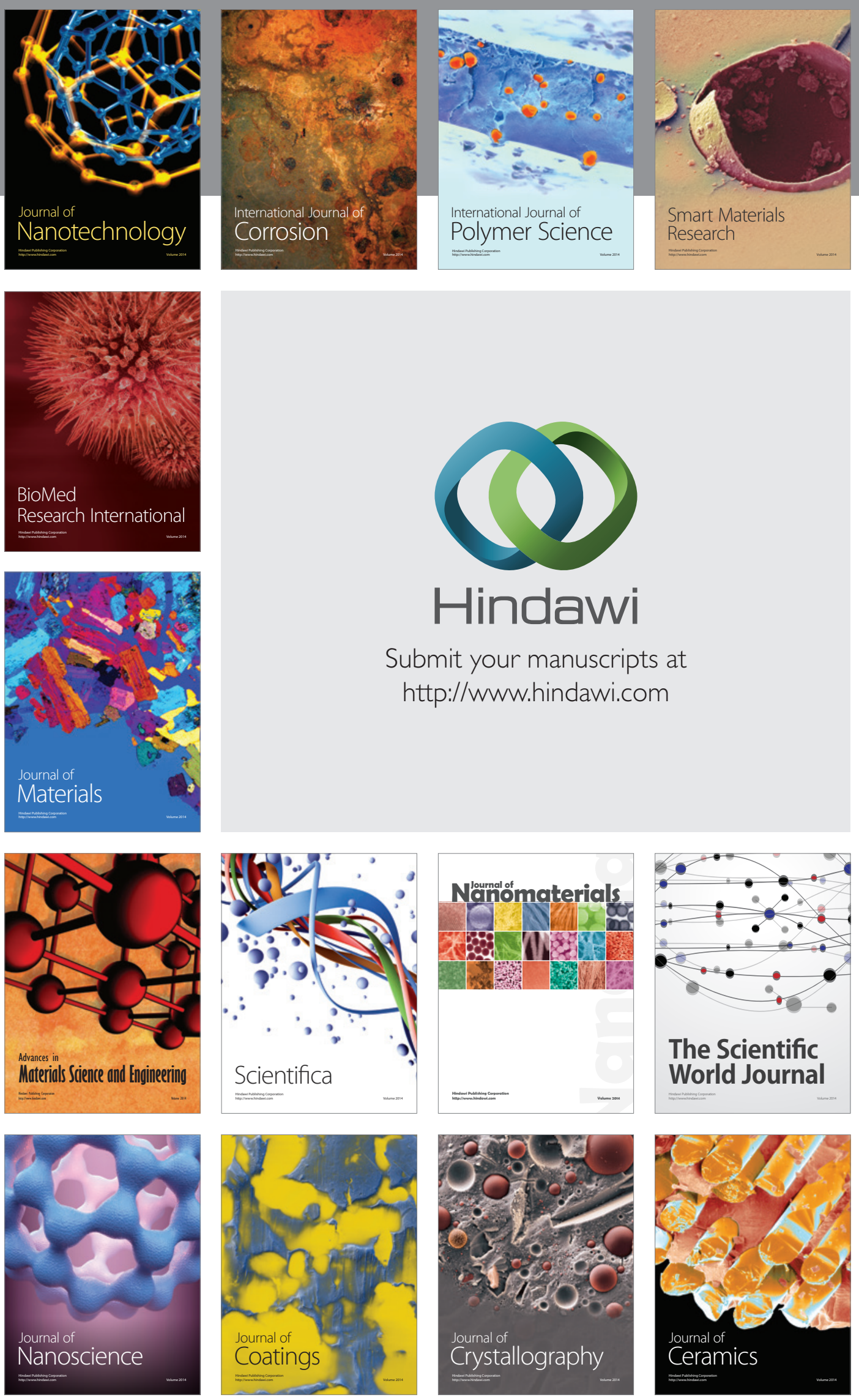

The Scientific World Journal

Submit your manuscripts at

http://www.hindawi.com

\section{World Journal}

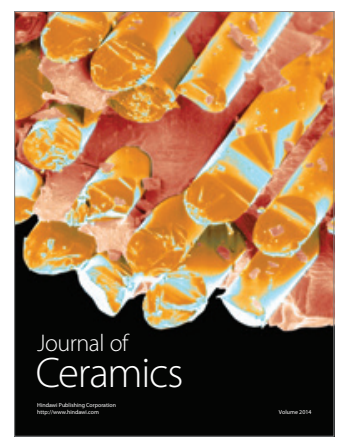

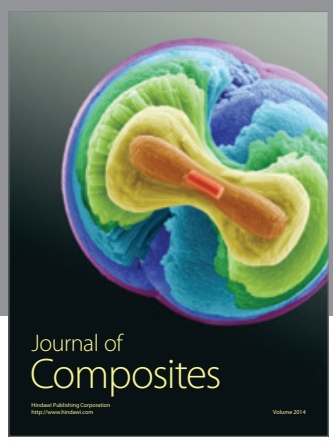
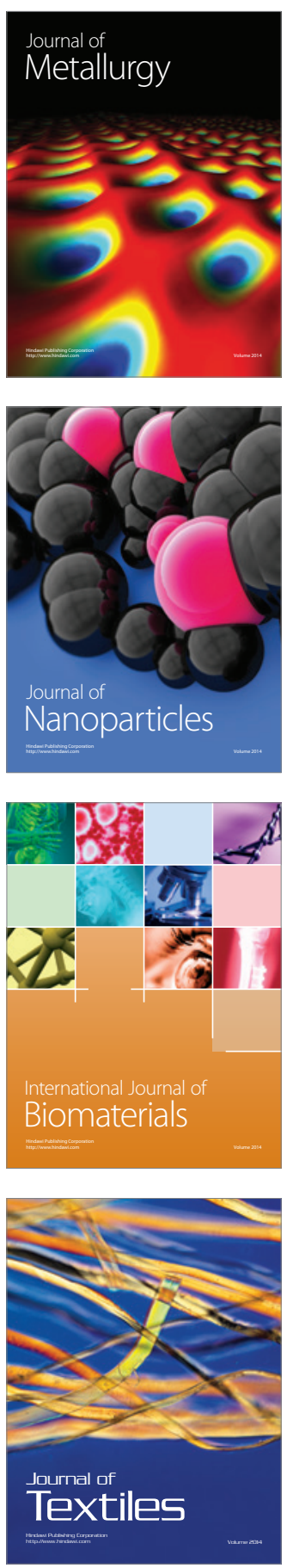